\section{Riding along on the crest of a wave}

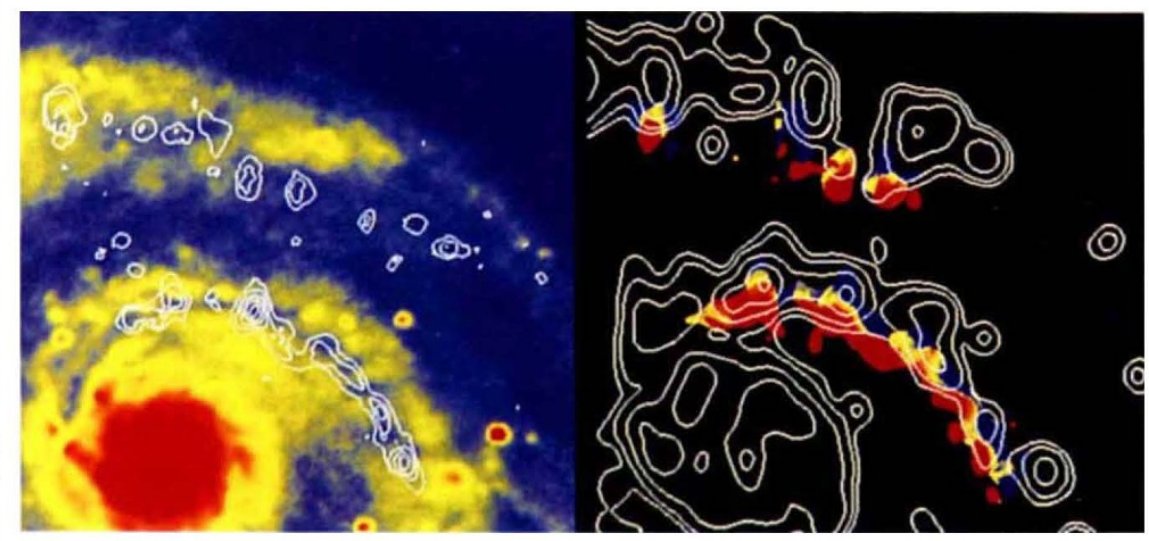

THE familiar spiral structure of many galaxies has more than visual appeal: gas and dust, the debris of stellar evolution, accumulate in the spiral arms to form a new generation of stars. But how spiral structure forms, and how it influences or perhaps controls star formation is not clear.

In the theory of density waves, spiral arms result from collective gravitational motions in the galactic disk, and make a travelling wave that orbits the disk. But not all galaxies have the perfect curved spokes of 'grand design' spirals. Often the arms are fragmented, leading some astrophysicists to suggest that they are purely local phenomena, perhaps the result of supernovae blowing out shells of material which get stretched into arcs by the differential rotation of the galaxy. Star formation happens as the supernova shock compresses existing clouds of gas, and the spiral arms are a consequence, not a cause, of star formation.

On page 402 of this issue, S.N. Vogel et al. present observations which favour the density-wave theory. On the left, contours representing the intensity of $\mathrm{CO}$ emission are superposed on an infrared image of the galaxy M51, showing that dense molecular clouds coincide with dust lanes in the spiral arms.

On the right, the colours represent the line-of-sight velocity of the $\mathrm{CO}$ emission relative to the velocity of the galaxy (red means motion towards us, blue away) and show a sharp change in velocity as the gas moves across the spiral arm. The contours are of hydrogen emission, associated with young stars; stars seem to be forming just downstream of gas that has passed through the density wave. These observations supply a consistent picture of molecular clouds being triggered into star formation as the density wave passes.

\section{Cosmic rays}

\section{More missing neutrinos}

\section{A.W. Wolfendale}

Protons may be reticent about decaying in public, but the detectors built to search for their decays are providing fascinating science. Coming hard on the heels of the first detection of neutrinos from supernova 1987A in the Large Magellanic Cloud in February last year, the Kamiokande group in Japan now reports a result which, if true, has an interpretation more important still. Hirata et al.' claim that their 3,000-ton detector located $1,000 \mathrm{~m}$ underground has detected too few muons produced by neutrinos interacting inside it, these neutrinos having been produced in the atmosphere by incoming cosmic-ray particles. Electrons produced in the detector by a similar process seem to appear at the expected rate, however.
As the authors conclude: "some as-yetunaccounted-for physics such as neutrino oscillations might explain the data". The Holy Grail of neutrino physicists - neutrino oscillations - might be at hand.

The neutrino ('the little neutral one') was postulated by Pauli to solve the problem of the missing energy in nuclear $\beta$-decay, so that the basic reaction producing $\beta$-particles (electrons) became neutron $\rightarrow$ proton + electron + neutrino or, more precisely, $\mathrm{n} \rightarrow \mathrm{p}+\mathrm{e}^{-}+\bar{v}_{\mathrm{e}}$, the bar indicating that the neutrino is in fact an antineutrino. The inverse reaction is $v_{\mathrm{e}}+\mathrm{n} \rightarrow \mathrm{p}+\mathrm{e}^{-}$and there is the equivalent reaction with protons: $\bar{v}_{\mathrm{e}}+\mathrm{p} \rightarrow \mathrm{n}+\mathrm{e}^{+}$.

This last-mentioned is the process detected experimentally by Reines and Cowan in the 1950 s, leading to the universal acceptance of the neutrino (of no mass and no charge) as a bona fide particle. It was discovered later that there are other neutrinos too - most importantly here, the muon neutrino, generated in the decay of pions: $\pi^{+} \rightarrow \mu^{+}+v_{\mu}$. The corresponding inverse reaction creates muons: $v_{\mu}+$ $\mathrm{n} \rightarrow \mathrm{p}+\mu^{-}$and it is this reaction that is the basis of the present excitement.

The search for muons produced by cosmic-ray neutrinos started in the early 1960 s when the first deep-mine experiments commenced with what were then

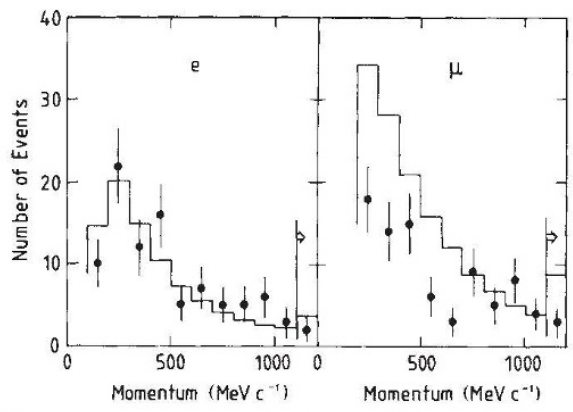

Momentum spectra of electron-like and muonlike events observed in the Kamiokande experiment $t^{1}$. The histograms are the expectations for electrons and muons generated by the interactions of neutrinos, the neutrinos themselves having come from the interactions of cosmic rays in the atmosphere.

very big detectors. Our group working in the Kolar Gold Fields ${ }^{2}$ and another in South Africa ${ }^{3}$ detected the underground muons, some of which were moving upwards and thus must have been generated by neutrino scattering (at somewhat below the correct rate). A hypothetical particle of interest then was the intermediate vector boson' and a lower limit of 3 gigaelectron volts $(\mathrm{GeV})$ was put on its mass from these results; as is well known, its discovery had to wait until the recent experiments at CERN led by Rubbia, and its mass is in fact nearly $90 \mathrm{GeV}$.

The search for the decay of the proton, expected on the basis of the grand unified theory, led to an upsurge in underground physics, and the Kamiokande experiment is one of two large-scale projects (the US IMB experiment is the other). It comprises a 3,000-ton water Cerenkov detector located in the Kamioka mine in Japan and has been in operation since July 1983. Particles produced in the water tank are detected by the Cerenkov radiation (electromagnetic 'shock waves') they produce and the pattern of the radiation leads to particle identification, albeit with some uncertainty. Hirata et al. ${ }^{1}$ use Monte Carlo calculations to derive the expected number of electron-like and muon-like events (see figure). Clearly, if the electronlike events are indeed electrons, and similarly for the muons, then there is a deficit of muons whereas the number of electrons is just about correct. 